\title{
Tunable Phononic Band Gaps of Surface and Bulk Acoustic Waves in Two-dimensional Phononic Crystals
}

\author{
Tsung-Tsong $\mathrm{Wu}$ \\ Institute of Applied Mechanics, \\ National Taiwan University, \\ Taipei 106, TAIWAN \\ wutt@ndt.iam.ntu.edu.tw
}

\author{
Zi-Gui Huang \\ Institute of Applied Mechanics, \\ National Taiwan University, \\ Taipei 106, TAIWAN \\ huang@ndt.iam.ntu.edu.tw
}

\begin{abstract}
In this paper, we present the results of the temperature effect on two-dimensional phononic crystals. Band gap variations of both of the bulk modes and surface modes due to the changing of temperature of the band structure in air/quartz band structure from zero to fifty degrees centigrade are calculated and discussed. The results show that the elastic band gaps can be enlarged or reduced by adjusting the temperature of the band structure. The temperature effect can potentially be utilized for fine-tuning of the phononic band gap frequency and the precise design of filters.
\end{abstract}

Keywords-phononic crystal; band gap; SAW;BAW

\section{INTRODUCTION}

The existence of complete band gaps of electromagnetic waves in photonic structures extending throughout the Brillouin zone has demonstrated a variety of fundamental and practical interests. Successful application of photonic crystals has led to rapid growing interests in the analogous acoustic effects in periodic elastic structures called the phononic crystals. Research in band gaps of bulk acoustic waves (BAW) of phononic structures have been carried out in the past [1-6]. The dispersion relations and band gap properties of the transverse polarization modes for periodic, elastic composites were analyzed and discussed in the past [1-3]. The dispersion relations of mixed polarization modes and the experimental evidence for the existence of absolute acoustic band gaps have also been investigated $[4,5]$. The effects of the orientation of square rods on the acoustic band gaps in a two-dimensional phononic crystal (solid/air) were discussed [6,7]. In [8-11], the multiple scattering theory was applied to study the band gaps of bulk wave in three-dimensional periodic acoustic composites and the band structure of a phononic crystal consisting of complex and frequency dependent Lame' coefficients. The finite difference time domain method was applied to interpret the experimental data of two-dimensional systems consisting of cylinders of fluids inserted periodically in a finite slab of Al host [12].

Investigations into surface wave (SAW) properties of solids in which the periodic modulation occurs on the traction-free surface did not take place until quite recently [13-15]. References 13 and 14 reported the calculations for surface waves on a square and hexagonal superlattice, consisting of cubic $(\mathrm{AlAs} / \mathrm{GaAs})$ and isotropic $(\mathrm{Al} /$ polymer) materials, and $\mathrm{Wu}$ et al [15] extended these works by studying the phononic band gaps of the SAW and BAW modes in two-dimensional phononic structures consisting of general anisotropic materials.

In this paper, we focus on the temperature effect for different polarization propagation modes in two-dimensional periodic structures. The plane wave expansion (PWE) method was adopted in [15] to calculate the variations of band gap widths of the quasi-shear vertical (SV), quasi-shear horizontal (SH), quasi-longitudinal (L) and surface acoustic wave (SAW) modes due to the temperature changes.

\section{THEORY}

A brief introduction of the theory is given in the following. In an inhomogeneous linear elastic medium with no body force, the equation of motion of the displacement vector $\mathbf{u}(\mathbf{r}, t)$ can be written as

$$
\rho(\mathbf{r}, T) \ddot{u}_{i}(\mathbf{r}, t)=\partial_{j}\left[C_{i j m n}(\mathbf{r}, T) \partial_{n} u_{m}(\mathbf{r}, t)\right],
$$

where $\mathbf{r}=(\mathbf{x}, z)=(x, y, z)$ is the position vector, $T$ is the temperature variable, $t$ is the time variable; $\rho(\mathbf{r}, T)$ and $C_{i j m n}(\mathbf{r}, T)$ are the position-dependent and temperaturedependent mass density and elastic stiffness tensor respectively. In the following, we consider a phononic crystal composed of a two-dimensional periodic array ( $x-y$ plane) of material $A$, embedded in a background material $B$. Due to the spatial periodicity, the material constants, $\rho(\mathbf{x}, T)$ and $C_{i j m n}(\mathbf{x}, T)$ can be expanded at temperature $T$ and in Fourier series, with respect to the two-dimensional reciprocal lattice vectors (RLV), $\mathbf{G}=\left(G_{1}, G_{2}\right)$, as

$$
\begin{aligned}
& \rho(\mathbf{x}, T)=\sum_{\mathbf{G}} e^{i \mathbf{G} \cdot \mathbf{x}} \rho_{\mathbf{G}}(T), \\
& C_{i j m n}(\mathbf{x}, T)=\sum_{\mathbf{G}} e^{i \mathbf{G} \cdot \mathbf{x}} C_{\mathbf{G}}^{i j m n}(T),
\end{aligned}
$$


where $\rho_{\mathbf{G}}(T)$ and $C_{\mathbf{G}}^{i j m n}(T)$ are the corresponding Fourier coefficients.

To utilize the Bloch's theorem and to expand the displacement vector $\mathbf{u}(\mathbf{r}, t)$ in Fourier series for the analyses of the surface and bulk waves, we have

$$
\mathbf{u}(\mathbf{r}, t)=\sum_{\mathbf{G}} e^{i \mathbf{k} \cdot \mathbf{x}-i \omega t}\left(e^{i \mathbf{G} \cdot \mathbf{x}} \mathbf{A}_{\mathbf{G}} e^{i k_{z} z}\right),
$$

where $\mathbf{k}=\left(k_{1}, k_{2}\right)$ is the Bloch wave vector, $\omega$ is the circular frequency, $k_{z}$ is the wave number along the $z$ direction, and $\mathbf{A}_{\mathbf{G}}$ is the amplitude of the displacement vector. We note that as the component of the wave vector $k_{z}$ equals zero, equation (4) degenerates into the displacement vector of a bulk acoustic wave. On substituting equations (2), (3) and (4) into equation (1), and after collecting terms systematically, we obtain the generalized eigenvalue problem as

$$
\left(\mathbf{A} k_{z}^{2}+\mathbf{B} k_{z}+\mathbf{C}\right) \cdot \mathbf{U}=0
$$

where $\mathbf{A}, \mathbf{B}$ and $\mathbf{C}$ are $3 n \times 3 n$ matrices, and are functions of the Bloch wave vector $\mathbf{k}$, components of the two-dimensional RLV, circular frequency $\omega$, the Fourier coefficients of mass density $\rho_{\mathbf{G}}(T)$ and components of elastic stiffness tensor $C_{\mathbf{G}}^{i j m n}(T) . \quad n$ is the total number of RLV used in the Fourier expansion, and $\mathbf{U}$ is the eigenvector. The expressions of the matrices $\mathbf{A}, \mathbf{B}$ and $\mathbf{C}$ were listed in [15].

By applying the surface wave conditions and the traction free boundary conditions on the surface, the dispersion relation for the surface waves propagating in the two-dimensional phononic crystals, with both of the filling and background materials belonging to the triclinic system, can be obtained [15]. When $k_{z}$ in equation (5) is equal to zero, the equation degenerates into the eigenvalue problem of the bulk waves as

$$
\mathbf{C} \cdot \mathbf{U}=0 .
$$

The dispersion relations of the bulk waves propagating in the two-dimensional phononic crystals can be obtained by setting the determinant of matrix $\mathbf{C}$ equal to zero. At the same time, for materials with symmetry lower than orthorhombic symmetry, the matrix $\mathbf{C}$ can not be decoupled into two different polarization modes (mixed and transverse polarization modes) [15]. The full matrix $\mathbf{C}$ must be considered, and distinguished as quasi-SV, quasi-SH and quasi-L modes.

\section{AIR/QUARTZ BAND STRUCTURE}

Consider phononic structures consisted of circular cylinders and a background material forming a two-dimensional square lattice with lattice spacing $a(=10 \mu \mathrm{m})$ shown in Fig. 1. Air and quartz are utilized as both of the cylinders and the background materials respectively. From the elastic constants and density at room temperature $\left(25^{\circ} \mathrm{C}\right)$, the temperature-dependent elastic constants and densities of the material, quartz, can be obtained by using [16]

$$
X(T) \approx X\left(T_{0}\right)\left[1+\frac{1}{X\left(T_{0}\right)} \frac{\partial X}{\partial T}\left(T-T_{0}\right)+\frac{1}{2 X\left(T_{0}\right)} \frac{\partial^{2} X}{\partial T^{2}}\left(T-T_{0}\right)^{2}+\ldots\right],
$$

where $T_{0}$ is $25^{\circ} \mathrm{C}$, and $X\left(T_{0}\right)$ is the elastic constant evaluated at room temperature. $\frac{1}{X\left(T_{0}\right)} \frac{\partial X}{\partial T}$ and $\frac{1}{2 X\left(T_{0}\right)} \frac{\partial^{2} X}{\partial T^{2}}$ are the first and the second order temperature coefficients respectively. $T$ is increased from $0^{\circ} \mathrm{C}$ to $50^{\circ} \mathrm{C}$. The first order temperature coefficient of the density reads

$$
\frac{1}{\rho\left(T_{0}\right)} \frac{\partial \rho}{\partial T}=-\left(\alpha_{11}+\alpha_{22}+\alpha_{33}\right),
$$

where $\alpha_{11}, \alpha_{22}$, and $\alpha_{33}$ are the expansion coefficients along the $x, y$, and $z$ axis respectively.

In this paper, we only considered the first order temperature coefficients and expansion coefficients of quartz (Z-cut). The related constants can be found in [17] and the thermodynamic properties (density and sound speed) of air can be found in [18]. It is worth noting that the filling fraction and the effects of the thermal stresses arising from the thermal expansion mismatch between the superlattice components when the temperature is varied are neglected due to the air/solid band structure.

The case considered in this paper is that we increase the temperature of the air/quartz band structure from zero to fifty degrees centigrade. The phononic crystal consists of quartz substrate and square arrays of air cylindrical holes. The modified PWE method can be applied because of the highdensity contrast between solid and air [6,7]. It is well-known that the high density contrast between solid and air leads to unexpected flat bands in the band structure. We get round this problem by adopting the technique shown in [6]. The flat bands can be removed by taking an artificial transverse velocity inside the fluid. Instead of postulating a purely longitudinal behavior of the solid [7], we also give an artificial transverse character to the fluid [6].

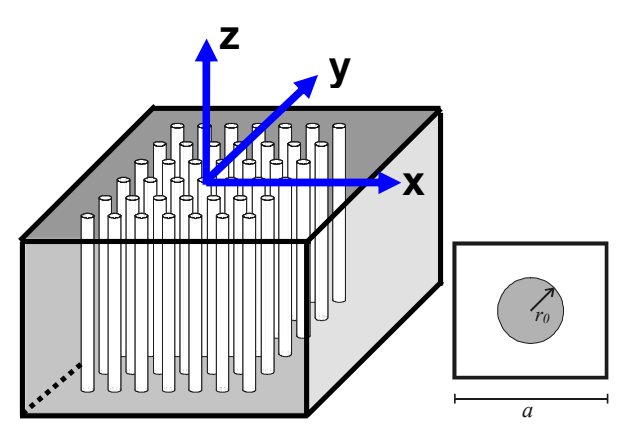

Figure 1. A two-dimensional square lattice with lattice spacing $a(=10 \mu \mathrm{m})$ 


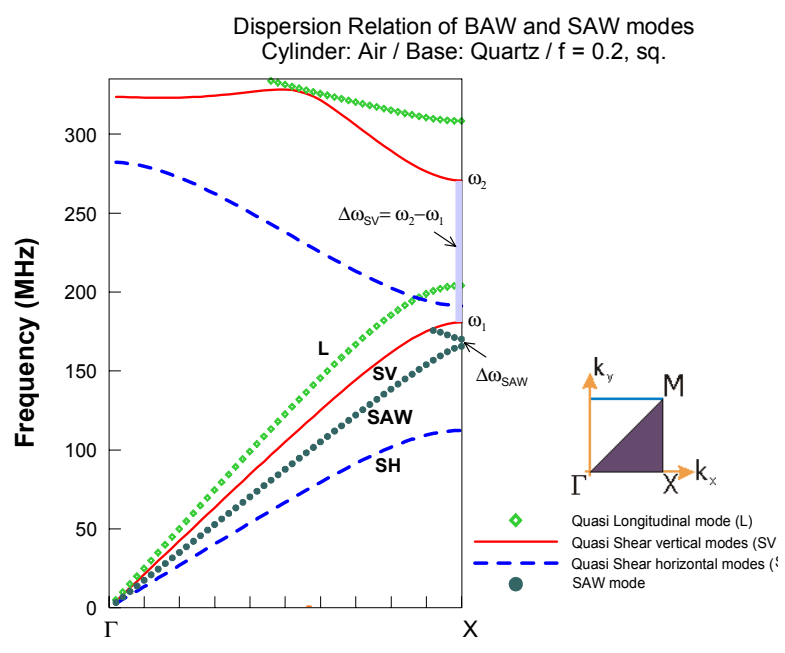

Figure 2. Dispersion relations of the quasi-SV, quasi-SH, quasi-L and SAW modes in air/quartz phononic structure with square lattice.

Fig. 2 shows the dispersion relations of the surface and bulk modes along the $\Gamma-\mathrm{X}$ section in the irreducible part of the Brillouin zone (see inset of Fig. 2) in air/quartz band structure with a filling ratio of 0.2 . In the calculations, the $x-y$ plane is parallel to the (001) plane and the $x$ axis is along the [100] direction of quartz, and the elastic properties and densities of the materials from $0^{\circ} \mathrm{C}$ to $50^{\circ} \mathrm{C}$ utilized in this example are calculated from equations (7) and (8). The vertical axis is the frequency in $\mathrm{MHz}$ unit and the horizontal axis is the reduced wave vector $k^{*}=k a / \pi . k$ is the wave vector along the Brillouin zone. The thin solid lines represent the fundamental and higher quasi-SV modes, and the square symbols represent the quasi- $\mathrm{L}$ modes. The thin dashed lines represent the quasiSH modes, while solid circles represent the surface acoustic modes. The band gap width is defined as $\Delta \omega=\omega_{2}-\omega_{1}\left(\omega_{1}\right.$, $\omega_{2}$ are the frequencies at the X point) and $\Delta \omega_{S V}, \Delta \omega_{S H}, \Delta \omega_{L}$, and $\Delta \omega_{S A W}$ represent the band gap widths for quasi-SV, quasi$\mathrm{SH}$, quasi-L and SAW modes respectively. At room
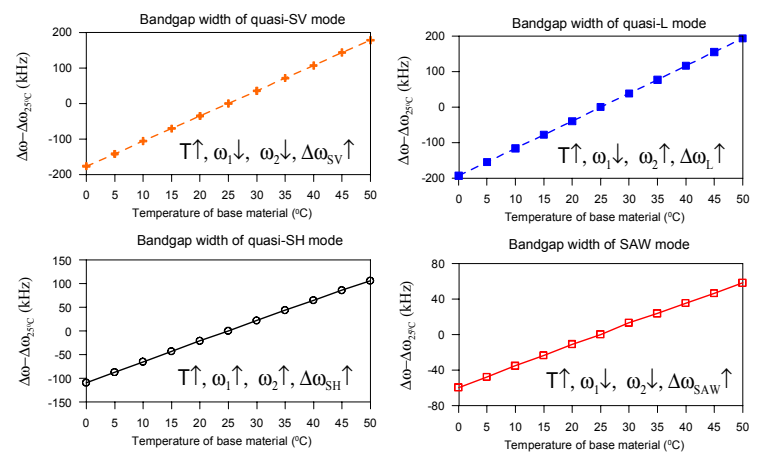

Figure 3. Band gap variations of air/quartz phononic structure with cylinder: air $\left(0^{\circ} \mathrm{C}-50^{\circ} \mathrm{C}\right)$ and base: quartz $\left(0^{\circ} \mathrm{C}-50^{\circ} \mathrm{C}\right)$ temperature, the band gap widths for the above four types of modes are $\Delta \omega_{S V}=89.98 \mathrm{MHz}, \Delta \omega_{S H}=78.04 \mathrm{MHz}, \Delta \omega_{L}=$ 104.76 MHz, and $\Delta \omega_{S A W}=4.77 \mathrm{MHz}$.

With the temperature of the band structure being increased from zero to fifty degrees centigrade, Fig. 3 shows the detail variations of the band gap widths of the four types of modes. The vertical axis is the frequency variation of the band gap defined as $\Delta \omega-\Delta \omega_{25^{\circ} \mathrm{C}}$ (unit: $\mathrm{kHz}$ ) and the horizontal axis is the temperature varying from $0^{\circ} \mathrm{C}$ to $50^{\circ} \mathrm{C}$. The results show that $\omega_{1}$ and $\omega_{2}$ of the quasi-SH mode at point X increase as the temperature rises, but those of the quasi-SV and SAW modes decrease. At point $X$, the $\omega_{1}$ of the quasi- $\mathrm{L}$ mode decreases while $\omega_{2}$ increases as the temperature rises. In Fig. 3, the relative variations of the band gap widths $\Delta \omega_{S V}, \Delta \omega_{S H}, \Delta \omega_{L}$ and $\Delta \omega_{S A W}$ all increase in direct proportion to the temperature change.

\section{CONCLUSION}

Some important conclusions can be drawn from the above studies. First, we found that the temperature effects are obvious for all types of modes in the air/quartz band structure. Secondly, the tendencies of the relative band gap widths for the four type modes are the same in air/quartz band structure while the variations of the frequencies at $\mathrm{X}$ point are quite different. The frequencies $\omega_{1}$ and $\omega_{2}$ would decrease as the temperature rises. However, the $\omega_{1}$ and $\omega_{2}$ of quasi-SH modes and the $\omega_{2}$ of quasi-L modes in the air/quartz band structure increase as the temperature rises. We note that the band gap width can be enlarged or reduced by adjusting the temperature of the band structure. These prominent features of the temperature effect on the band gap variations of the phononic crystals may have potential applications in fine-tuning of the phononic band gap and they are very important on the precise filter design.

\section{ACKNOWLEDGMENTS}

The authors gratefully acknowledge the National Science Council and the NTU-ITRI center of Taiwan for financial support.

\section{REFERENCES}

[1] M. S. Kushwaha, P. Halevi, L. Dobrzynski, and B. Djafari-Rouhani, "Acoustic Band Structure of Periodic Elastic Composites," Phys. Rev. Lett. 71, pp. 2022-2025, 1993.

[2] M. S. Kushwaha and P. Halevi, "Band-gap engineering in periodic elastic composites,” Appl. Phys. Lett. 64, pp. 1085-1087, 1994.

[3] M. S. Kushwaha, P. Halevi, G. Martinez, L. Dobrzynski, and B. DjafariRouhani, "Theory of acoustic band structure of periodic elastic composites," Phys. Rev. B 49, pp. 2313-2322, 1994.

[4] J. O. Vasseur, B. Djafari-Rouhani, L. Dobrzynski, M. S. Kushwaha, P. Halevi, "Complete acoustic band gaps in periodic fibre reinforced composite materials: the carbon/epoxy composite and some metallic systems," J. Phys.: Condens. Matter 6, pp. 8759-8770, 1994.

[5] J. O. Vasseur, P. A. Deymier, G. Frantziskonis, G. Hong, B. DjafariRouhani and L. Dobrzynski, "Experimental evidence for the existence of absolute acoustic band gaps in two-dimensional periodic composite media," J. Phys.: Condens. Matter 10, pp. 6051-6064, 1998. 


\section{IEEE Ultrasonics Symposium}

[6] Goffaux, C. and Vigneron, "Theoretical study of a tunable phononic band gap system," Phys. Rev. B 64, pp. 075118, 2001.

[7] Xiaoling Li, Fugen Wu, Hefei Hu, Shao Zhong and Youyan Liu, "Large acoustic band gaps created by rotating square rods in two-dimensional periodic composites," J. Phys. D: Appl. Phys. 36, pp. L15-L17, 2003.

[8] M. Kafesaki and E. N. Economou, "Multiple-scattering theory for threedimensional periodic acoustic composites," Phys. Rev. B 60, pp. 11993, 1999.

[9] I. E. Psarobas and N. Stefanou, "Scattering of elastic waves by periodic arrays of spherical bodies," Phys. Rev. B 62, pp. 278, 2000.

[10] Zhengyou Liu, C. T. Chan, and Ping Sheng, "Elastic wave scattering by periodic structures of spherical objects: Theory and experiment," Phys. Rev. B 62, pp. 2446, 2000.

[11] Jun Mei, Zhengyou Liu, Jing Shi, and Decheng Tian, “Theory for elastic wave scattering by a two-dimensional periodical array of cylinders: An ideal approach for band-structure calculations," Phys. Rev. B 67, pp. 245107, 2003.
[12] D. Garica-Pablos, M. Sigalas, F. R. Montero de Espinosa, M. Kafesaki and N. Garcia, "Theory and Experiments on Elastic Band gaps," Phys. Rev. Lett. 84, pp. 4349, 2000.

[13] Y. Tanaka and S. Tamura, "Surface acoustic waves in two-dimensional periodic elastic structures," Phys. Rev. B 58, pp. 7958, 1998

[14] Y. Tanaka and S. Tamura, "Acoustic stop bands of surface and bulk modes in two-dimensional phononic lattices consisting of aluminum and a polymer," Phys. Rev. B 60, 13 294, 1999.

[15] Tsung-Tsong Wu, Zi-Gui Huang and S. Lin, "Surface and bulk acoustic waves in two-dimensional phononic crystals consisting of materials with general anisotropy," Phys. Rev. B 69, pp. 094301, 2004.

[16] A. A. Oliner, Acoustic Surface Waves-topics in Applied Physics 24, Springer-Verlag Berlin Heidelberg, New York, 1978.

[17] M. B. Schulz, B. J. Matsinger, and M. G. Holland, "Temperature dependence of aurface acoustic wave velocity on $\alpha$ quartz," J. Appl. Phys. 41, pp. 2755, 1970.

[18] David R. Lide, CRC handbook of chemistry and physics, CRC press 83rd ed. Boca Raton London, NY, 2002-2003. 